\title{
Breakdown of the classical double copy for the effective action of dilaton-gravity at NNLO
}

\author{
Jan Plefka $\odot,{ }^{1, *}$ Canxin Shi, ${ }^{1}$ Jan Steinhoff $\odot,{ }^{2}$ and Tianheng Wang ${ }^{1}$ \\ ${ }^{1}$ Institut für Physik und IRIS Adlershof, Humboldt-Universität zu Berlin, \\ Zum Großen Windkanal 6, 12489 Berlin, Germany \\ ${ }^{2}$ Max-Planck-Institut für Gravitationsphysik (Albert-Einstein-Institut), \\ Mühlenberg 1, D-14476 Potsdam, Germany
}

(Received 7 August 2019; published 11 October 2019)

\begin{abstract}
We demonstrate that a recently proposed classical double-copy procedure to construct the effective action of two massive particles in dilaton-gravity from the analogous problem of two color-charged particles in Yang-Mills gauge theory fails at next-to-next-to-leading order perturbative expansions, i.e., in the third order of the post-Minkowskian and the second order in the post-Newtonian expansions.
\end{abstract}

DOI: 10.1103/PhysRevD.100.086006

\section{INTRODUCTION AND CONCLUSIONS}

A spectacular success in fundamental physics has been the detection of gravitational waves at the LIGO/Virgo detectors since 2015 [1]. This detection and analysis hinges crucially on waveform templates emerging from high-precision theoretical predictions in general relativity including higher-order perturbative computations of the two-body effective potential in the post-Newtonian $(\mathrm{PN})$, i.e., weak gravitational field and slow motion, and post-Minkowskian (PM), i.e., weak gravitational field, regimes. These predictions build on established perturbative formalisms in general relativity [2-6] as well as on the effective field theory approach $[7,8]$ yielding a quantum field theoretical diagrammatic expansion for the classical effective potential (for introductory reviews see [9-11]). The present state of the art in the PN expansion is the conservative effective potential at 4PN [12-18], at 5PN for the static part $[19,20]$ as well as 3PN [21] for the gravitational radiation emitted from a quasicircular inspiral. In particular the effective-one-body waveform model [22-25] relies on accurate theoretical predictions for the potential of the binary.

In the past decade important progress in the study of scattering amplitudes in gauge theories and gravity occurred based on innovative perturbative on-shell techniques and discovered mathematical structures that greatly increased the ability to compute gravitational

jan.plefka@physik.hu-berlin.de

Published by the American Physical Society under the terms of the Creative Commons Attribution 4.0 International license. Further distribution of this work must maintain attribution to the author(s) and the published article's title, journal citation, and DOI. Funded by SCOAP. scattering amplitudes at high perturbative orders. In particular the efficient double-copy construction based on the Bern-Carrasco-Johansson color-kinematical duality [26-28] yields the integrands of gravitational scattering amplitudes from the simpler quantities in Yang-Mills theories, allowing for high-order results in (super)gravity (see, e.g., the recent $[29,30]$ ). In view of these innovations it is natural to ask to what extent these modern scattering amplitude techniques may be put to work to the classical scattering problem in general relativity and in turn to the perturbative construction of effective potentials discussed above.

The question how the classical two-body gravitational potential may be extracted from the quantum scattering amplitude of (say) massive scalars has a long history [31-33]. Recent works have updated these results by employing the above-mentioned modern unitarity methods for amplitudes [34-38], also including higher curvature terms [39,40], leading to the 2PM [41,42] and the first 3PM [43] results for the effective gravitational potential very recently. A 2PM Hamiltonian matched to the classical scattering angle [6] was obtained in $[44,45]$.

In parallel there are indications for the existence of a classical double copy of gauge theory to gravity beyond the realm of scattering amplitudes. In a series of works [46-51] a selection of gravitational solutions were shown to be double copies of Yang-Mills ones. Relevant for the problem of classical gravitational radiation has been the approach of Goldberger and Ridgway [52] generating perturbative solutions to the equations of motion for a binary pair of spinless massive particles in dilaton-gravity via the double copy of binary color-charged point particle solutions in Yang-Mills theory [53-57]. Here color-kinematic replacement rules were employed and refined in [58], thereby pushing this perturbative double-copy technique to the 
next-to-next-to-leading order in the coupling constant expansion. In these works a certain challenge lies in the clean identification of the kinematic numerators and propagators in order to perform the double copy in analogy to the procedure known from scattering amplitudes, where a factorization into color factors, kinematic numerators and propagators is manifest. This approach was lifted from the level of equations of motion to the effective action by Wormsbecher and two of the present authors [59] recently. Here an adapted version of the double-copy construction was presented that enabled a direct computation of the classical effective action for two massive particles in dilaton-gravity from the corresponding quantity for color-charged particles coupled to the Yang-Mills gauge field. It was shown that this modified prescription to compute the gauge dependent and off-shell effective potential yields the known result in dilaton-gravity [60] at 2PM level (at the integrand level) as well as 1PN (explicitly) in a much simpler fashion than the traditional approaches in general relativity. This proof of principle thus led us to the hope that it could provide a highly efficient tool to perform higher-order perturbative computations within gravity in a systematic fashion. This motivated the present work to push this expansion to the next order, i.e., 3PM (at the integrand level) and 2PN (exactly), respectively.

Unfortunately, we have to report that the effective action generated by the proposed double-copy procedure of [59] fails to agree with the desired dilaton-gravity result at this next-to-next-to-leading order. The dilatongravity potential at $2 \mathrm{PN}$ we sought to reproduce is available in the literature [61] and we have also checked its static contributions from a probe limit while comparing to the Janis-Newman-Winicour (JNW) naked singularity [62] as well as through a direct Feynman diagrammatic computation in order to be certain of the discrepancy. It is important to stress that this breakdown of the double-copy procedure applies to a gauge variant and off-shell quantity - the effective action. This might be the root of the breakdown. Similar problems should arise at higher orders for the double-copy construction of perturbative spacetimes going beyond the order considered in [48]. Note that there as well a comparison to the JNW solution occurs, which failed in our scenario. It is also conceivable that the double copy for scattering amplitudes involving massive external particles breaks down at the considered order (the leading order works fine [36]). If this is the case, then problems would also appear for the classical double copy at the next perturbative order for the emitted radiation at the level of the equations of motion [i.e., the order beyond [58] which is equivalent to the effective action at next-to-leading order (NLO) [59] plus an emitted gluon/graviton accounting for an additional factor of the coupling constant]. These interesting questions are left for future work.

\section{THE CLASSICAL DOUBLE COPY FOR THE EFFECTIVE POTENTIAL AT LEADING ORDERS IN PM}

The double copy of pure Yang-Mills theory is the massless sector of bosonic string theory defined by the action

$$
S_{\mathrm{dg}}=-\frac{2}{\kappa^{2}} \int d^{4} x \sqrt{-g}\left[R-2 \partial_{\mu} \phi \partial^{\mu} \phi\right],
$$

where $\kappa=m_{\mathrm{Pl}}^{-1}=\sqrt{32 \pi G}$ is the gravitational coupling (with Newton's constant $G$ and Planck mass $m_{\mathrm{Pl}}$ ), $\phi$ is a real scalar field known as the dilaton. The theory in question also contains an axion field which will be, however, irrelevant for our considerations. The worldline action of a point mass $m$ moving along its worldline trajectory $x^{\mu}(\tau)$ reads in the first-order formalism (we employ the conventions of [59])

$$
S_{\mathrm{pm}}=-\int d \tau\left(p_{\mu} \dot{x}^{\mu}-\lambda(\tau)\left[e^{-2 \phi} g^{\mu \nu} p_{\mu} p_{\nu}-m^{2}\right]\right),
$$

where $\lambda$ is a Lagrange multiplier. The effective potential for two point masses $m$ and $\tilde{m}$ may be computed in a weakfield (or post-Minkowskian) expansion by perturbing the metric around a flat Minkowski background $g_{\mu \nu}(x)=$ $\eta_{\mu \nu}+\kappa h_{\mu \nu}(x)$ and perturbatively integrating out the graviton field $h_{\mu \nu}$ and the dilaton $\phi$ in the path integral. This yields the effective action $S_{\text {eff,dg }}$

$$
\begin{aligned}
e^{\frac{i}{\hbar} S_{\text {eff,dg }}} & =e^{\frac{i}{\hbar} S_{\text {pm,free }}} \mathcal{M}_{\mathrm{dg}} \\
& =c \cdot \int \mathcal{D} h \mathcal{D} \phi e^{\frac{i}{\hbar}\left(S_{\mathrm{dg}}+S_{\mathrm{gf}}+S_{\mathrm{pm}}+\tilde{S}_{\mathrm{pm}}\right)},
\end{aligned}
$$

using a suitable gauge fixing term $S_{\mathrm{gf}}$. Here the normalization constant $c$ is chosen such that $\mathcal{M}_{\mathrm{dg}}=1$ for $\kappa \rightarrow 0$. $S_{\mathrm{pm}, \text { free }}$ is the sum of the worldline actions for masses $m$ and $\tilde{m}$ of Eq. (2) for $g_{\mu \nu}=\eta_{\mu \nu}$ and $\phi=0$. At leading order in $\kappa$ it is easy to see that $\mathcal{M}_{\mathrm{dg}}$ takes the form

$$
\tau_{1} \approx \approx \tilde{\tau}_{1}=-\frac{i \kappa^{2}}{\hbar} \int d \hat{\tau}_{1 \tilde{1}}\left(p_{1} \cdot \tilde{p}_{1}\right)^{2} D_{1 \tilde{1}},
$$

with the four-dimensional scalar propagator $D_{i j}:=$ $D\left(x_{i}-x_{j}\right)$, abbreviating $x_{i}=x\left(\tau_{i}\right)$ and with $\square D(x-y)=$ $-\delta(x-y)$. Moreover, we denote $d \hat{\tau}:=\lambda(\tau) d \tau$, as well as $d \hat{\tau}_{1 \tilde{1}}:=d \hat{\tau}_{1} d \hat{\tau}_{\tilde{1}}$.

The double-copy counterpart to Eq. (2) in Yang-Mills theory is a color-charged point particle moving along its worldline $x^{\mu}(\tau)$ with color charge $c^{a}(\tau)=\Psi^{\dagger}(\tau) T^{a} \Psi(\tau)$ where $\Psi(\tau)$ is a scalar worldline field. It couples to the gauge field $A_{\mu}^{a}(x)$ with strength $g$ through the first-order action (for details see [59]) 


$$
\begin{aligned}
S_{\mathrm{pc}}= & -\int d \tau\left(p_{\mu} \dot{x}^{\mu}-i \psi^{\dagger} \dot{\psi}\right. \\
& \left.-\lambda\left[p^{2}+2 g p_{\mu} A_{a}^{\mu} c^{a}+g^{2} A_{\mu}^{b} c^{b} A_{a}^{\mu} c^{a}-m^{2}\right]\right) .
\end{aligned}
$$

Note that the gauge field couples at most quadratically to the worldline, whereas the graviton has all order couplings in Eq. (2). In complete analogy to the gravitational case we define the effective potential for the color charge $S_{\text {eff,YM }}$ by integrating out the gluon field in the path integral via

$$
\begin{aligned}
e^{\frac{i}{\hbar} S_{\text {eff,YM }}} & =e^{\frac{i}{\hbar} S_{\text {eff, free }}} \mathcal{M}_{\mathrm{YM}} \\
& =c^{\prime} \cdot \int \mathcal{D} A e^{\frac{i}{\hbar}\left(S_{\mathrm{YM}}+S_{\mathrm{gf}}+S_{\mathrm{pc}}+\tilde{S}_{\mathrm{pc}}\right)} .
\end{aligned}
$$

The leading-order term in $g$ for $\mathcal{M}_{\mathrm{YM}}$ takes the form

$$
\tau_{1} \leadsto \tilde{\tau}_{1}=\frac{4 i g^{2}}{\hbar} \int d \hat{\tau}_{1 \tilde{1}}\left(c_{1} \cdot \tilde{c}_{1}\right)\left(p_{1} \cdot \tilde{p}_{1}\right) D_{1 \tilde{1}}
$$

Comparing this with Eq. (3) the double-copy structure is obvious: Replacing the color factor $\left(c_{1} \cdot \tilde{c}_{1}\right)$ with the kinematical numerator $\left(p_{1} \cdot \tilde{p}_{1}\right)$ in Eq. (7) along with the coupling replacement $2 g \rightarrow i \kappa$ yields the leading-order (LO) contribution to the dilaton-gravity effective potential.

In [59] it was shown that the double-copy prescription extends to NLO, i.e., $(2 g)^{4} \rightarrow(i \kappa)^{4}$. Here the relevant graph topologies on the Yang-Mills side are collected in Fig. 1.

The double-copy procedure proposed in [59] amounts to the following steps:

(1) Transform the Yang-Mills (YM) graphs to a trivalent structure: For the double gluon couplings to the worldline this amounts to the replacement

$$
\begin{aligned}
& \tau_{1} \underset{\sim}{\sim} \sim_{a \mu}^{b \nu} \rightarrow \delta\left(\tau_{1}-\tau_{2}\right) \cdot{ }_{\tau_{1}}^{\tau_{2}} \underset{\sim}{\sim}{ }^{b \nu} \mu \\
& \frac{2 i g^{2}}{\hbar} \int d \hat{\tau}_{1} c^{a}\left(\tau_{1}\right) c^{b}\left(\tau_{1}\right) \eta^{\mu \nu} \longrightarrow \\
& \frac{2 i g^{2}}{\hbar} \int d \hat{\tau}_{12} \frac{\delta\left(\tau_{1}-\tau_{2}\right)}{\lambda\left(\tau_{2}\right)} c^{a}\left(\tau_{1}\right) c^{b}\left(\tau_{2}\right) \eta^{\mu \nu} .
\end{aligned}
$$

In addition, one should seek a color-kinematic duality respecting representation of the bulk graphs dissolving the four-gluon vertex into three-gluon ones. However, this only

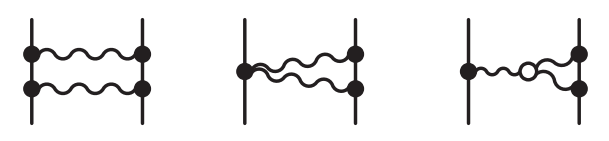

FIG. 1. Relevant graphs at NLO for the YM effective action. arises at the next-to-next-to-leading-order (NNLO) level and will be discussed in the following.

(2) Replace color factors with kinematics: Having established the trivalent representation the resulting form of $\mathcal{M}_{\mathrm{YM}}$ takes the general form

$$
\mathcal{M}_{\mathrm{YM}}^{\mathrm{N}^{n} \mathrm{LO}}=(2 g)^{2 n} \sum_{I \in \Gamma_{n}} \int \prod_{i_{I}} d \hat{\tau}_{i_{I}} \int d^{4 l_{I}} x \frac{C_{I} N_{I}}{S_{I} D_{I}},
$$

where $\Gamma_{n}$ represents the set of trivalent graphs at the considered order in perturbation theory, $C_{I}$ the occurring distinct color factors, $D_{I}$ the associated propagators and $N_{I}$ the numerators, while $S_{I}$ is the symmetry factor of graph $\Gamma_{i}$. Importantly, we keep the $\hbar$ dependence in all expressions, i.e., propagators come with a factor of $\hbar / i$ whereas vertices carry a uniform factor of $i / \hbar$. Once such a representation is established the double copy is performed by simply replacing $C_{I} \rightarrow N_{i}$, i.e.,

$$
\mathcal{M}_{\mathrm{dg}}^{\mathrm{N} n} \mathrm{LO}=(i \kappa)^{2 n} \sum_{I \in \Gamma_{n}} \int \prod_{i_{I}} d \hat{\tau}_{i_{I}} \int d^{4 l_{I}} x \frac{N_{I} N_{I}}{S_{I} D_{I}},
$$

which should be the exponentiated effective action of dilaton-gravity.

(3) Establish classical effective action: To find the classical effective potential one takes the logarithm of $\mathcal{M}_{\mathrm{dg}}$ and sends $\hbar$ to zero, i.e.,

$$
\lim _{\hbar \rightarrow 0} \frac{\hbar}{i} \ln \left[\sum_{n=0}^{\infty} \mathcal{M}_{\mathrm{dg}}^{\mathrm{N}^{n} \mathrm{LO}}\right]=S_{\mathrm{eff}, \mathrm{dg}}-S_{\mathrm{pm}, \text { free }} .
$$

In this classical limit ill-defined terms arising from squaring $\delta$-functions in the $N_{I}$ [such as $\frac{\hbar}{i} \delta(0)$ ] are suppressed. It is expected that they cancel with other quantum contributions.

(4) Integrate or perform $P N$ expansion: Finally, in order to establish the PM potential one should perform the bulk and $\tau_{i}$ integrals. For the PN approximation one first takes this limit and thereafter integrates.

The resulting effective action from Eq. (9) was shown to agree with the result in the literature on scalar-tensor theories [60] up to and including 1PN order. Moreover, it was shown in [59] that the double-copy result also agrees at the $2 \mathrm{PM}$ order at the level of integrands.

\section{YANG-MILLS COMPUTATION AT NNLO}

We now turn to the discussion of the NNLO computation via the double copy. To begin with consider the group of the diagrams that, with the introduction of the appropriate delta functions in the sense of Eq. (8), share the color factor of the three ladder diagram, i.e., $(c \cdot \tilde{c})^{3}$. 


$$
\stackrel{\sim}{\sim \sim 1}=\frac{1}{6}\left(\frac{i}{\hbar}\right)^{3}(2 g)^{6} \int d \hat{\tau}_{123 \tilde{1} \tilde{2} \tilde{3}}\left(p_{1} \cdot \tilde{p}_{1}\right)\left(p_{2} \cdot \tilde{p}_{2}\right)\left(p_{3} \cdot \tilde{p}_{3}\right)\left(c_{1} \cdot \tilde{c}_{1}\right)\left(c_{2} \cdot \tilde{c}_{2}\right)\left(c_{3} \cdot \tilde{c}_{3}\right) D_{1 \tilde{1}} D_{2 \tilde{2}} D_{3 \tilde{3}}
$$

$$
\begin{aligned}
\underset{1 \sim 1}{\sim \sim}= & \frac{1}{6}\left(\frac{i}{\hbar}\right)^{3}(2 g)^{6} \int d \hat{\tau}_{123 \tilde{1} \tilde{2} \tilde{3}} \frac{1}{2} \frac{\hbar}{i}\left[\frac{\delta\left(\tau_{1}-\tau_{2}\right)}{\lambda_{2}}\left(\tilde{p}_{1} \cdot \tilde{p}_{2}\right)\left(p_{3} \cdot \tilde{p}_{3}\right)+\frac{\delta\left(\tau_{2}-\tau_{3}\right)}{\lambda_{3}}\left(\tilde{p}_{2} \cdot \tilde{p}_{3}\right)\left(p_{1} \cdot \tilde{p}_{1}\right)\right. \\
& \left.+\frac{\delta\left(\tau_{3}-\tau_{1}\right)}{\lambda_{1}}\left(\tilde{p}_{1} \cdot \tilde{p}_{3}\right)\left(p_{2} \cdot \tilde{p}_{2}\right)\right]\left(c_{1} \cdot \tilde{c}_{1}\right)\left(c_{2} \cdot \tilde{c}_{2}\right)\left(c_{3} \cdot \tilde{c}_{3}\right) D_{1 \tilde{1}} D_{2 \tilde{2}} D_{3 \tilde{3}},
\end{aligned}
$$

$$
\begin{aligned}
\approx \sim & =\frac{1}{6}\left(\frac{i}{\hbar}\right)^{3}(2 g)^{6} \int d \hat{\tau}_{123 \tilde{2} \tilde{2} \tilde{3}} \frac{1}{6}\left(\frac{\hbar}{i}\right)^{2}\left[\frac{\delta\left(\tau_{1}-\tau_{2}\right)}{\lambda_{2}} p_{3}^{\mu}+\operatorname{cyclic}(1,2,3)\right]\left[\frac{\delta\left(\tilde{\tau}_{1}-\tilde{\tau}_{2}\right)}{\tilde{\lambda}_{2}} \tilde{p}_{3}^{\nu}+\operatorname{cyclic}(\tilde{1}, \tilde{2}, \tilde{3})\right] \eta_{\mu \nu} \\
& \times\left(c_{1} \cdot \tilde{c}_{1}\right)\left(c_{2} \cdot \tilde{c}_{2}\right)\left(c_{3} \cdot \tilde{c}_{3}\right) D_{1 \tilde{1}} D_{2 \tilde{2}} D_{3 \tilde{3}}
\end{aligned}
$$

Note that there is also a mirrored graph to Eq. (11) obtained by swapping tilded and nontilded quantities. Also, we write the integrands always in a completely symmetrized fashion with respect to permutations of the $\tau_{i}$ and $\tilde{\tau}_{i}$ proper-time variables. This prescription has an impact on the double copy and guarantees exponentiation as we shall see. The double copy of these diagrams will have a nontrivial contribution at $2 \mathrm{PN}$.

The second group of diagrams shares one bulk vertex and has the common color factor $(c \cdot[\tilde{c}, \tilde{c}])(c \cdot \tilde{c})$ reading

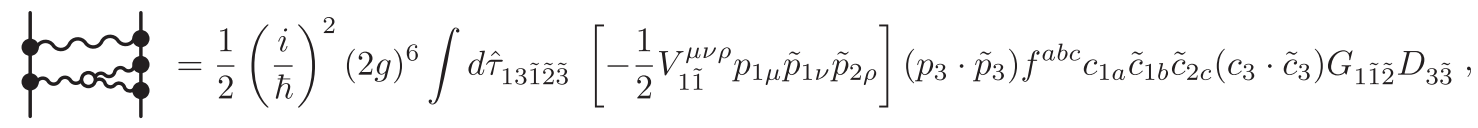

$$
\begin{aligned}
& \underset{1}{1}=\frac{1}{2}\left(\frac{i}{\hbar}\right)^{2}(2 g)^{6} \int d \hat{\tau}_{13 \tilde{1} \tilde{2} \tilde{3}} \frac{\hbar}{i}\left[-\frac{1}{4} \frac{\delta\left(\tilde{\tau}_{2}-\tilde{\tau}_{3}\right)}{\tilde{\lambda}_{3}} V_{1 \tilde{1}}^{\mu \nu \rho} \tilde{p}_{1 \nu}+(\tilde{1} \leftrightarrow \tilde{2})\right] p_{1 \mu} p_{3 \rho} f^{a b c} c_{1 a} \tilde{c}_{1 b} \tilde{c}_{2 c}\left(c_{3} \cdot \tilde{c}_{3}\right) G_{1 \tilde{1} \tilde{2}} D_{3 \tilde{3}} \\
& \underset{\sim}{\sim}=\frac{1}{2}\left(\frac{i}{\hbar}\right)^{2}(2 g)^{6} \int d \hat{\tau}_{13 \tilde{1} \tilde{2} \tilde{3}} \frac{\hbar}{i} \frac{\delta\left(\tau_{1}-\tau_{3}\right)}{\lambda_{3}} f^{a b c} c_{1 a} \tilde{c}_{1 b} \tilde{c}_{2 c}\left(c_{3} \cdot \tilde{c}_{3}\right)\left[-\frac{1}{4} V_{1 \tilde{1}}^{\mu \nu \rho} \tilde{p}_{3 \mu} \tilde{p}_{1 \nu} \tilde{p}_{2 \rho}\right] G_{1 \tilde{1} \tilde{2}} D_{3 \tilde{3}}
\end{aligned}
$$

where $V_{12}^{\mu \nu \rho}=\eta^{\mu \nu}\left(\partial_{1}-\partial_{2}\right)^{\rho}+\eta^{\nu \rho}\left(\partial_{1}+2 \partial_{2}\right)^{\mu}+\eta^{\rho \mu}\left(-2 \partial_{1}-\partial_{2}\right)^{\nu}$ and $G_{1 \tilde{1} \tilde{2}}=\int d^{4} x D_{1 x} D_{\tilde{1} x} D_{\tilde{2} x}$. It turns out that the double copy of these graphs is relevant to the 3PM, but does not contribute at the 2PN level. Here we only display their 3PM integrals and suppressed the mirror diagrams obtained by swapping the tilded and nontilded indices.

The third group of diagrams containing bulk vertices at order $g^{2}$ being symmetric with respect to both worldlines is characterized by the color structures $[c, c]^{a}[\tilde{c}, \tilde{c}]^{a}$ and $[c, \tilde{c}]^{a}[\tilde{c}, c]^{a}$. One now has

$$
\text { \{ron }=\frac{1}{4} \frac{i}{\hbar}(2 g)^{6} \int d \hat{\tau}_{12 \tilde{1} \tilde{2}} f^{a b e} f^{c d e} c_{1 a} c_{2 b} \tilde{c}_{1 c} \tilde{c}_{2 d}\left[\frac{1}{4} V_{12}^{\mu \nu \lambda} V_{\tilde{1} \tilde{2}}^{\rho \sigma \delta} \eta_{\lambda \delta} p_{1 \mu} p_{2 \nu} \tilde{p}_{1 \rho} \tilde{p}_{2 \sigma}\right] G_{12 ; \tilde{1} \tilde{2}}
$$




$$
\begin{aligned}
& \text { mond }=\frac{\xi}{2} \frac{i}{\hbar}(2 g)^{6} \int d \hat{\tau}_{12 \tilde{1} \tilde{2}} f^{a c e} f^{b d e} c_{1 a} c_{2 b} \tilde{c}_{1 c} \tilde{c}_{2 d}\left[\frac{1}{4} V_{1 \tilde{1}}^{\mu \rho \lambda} V_{2 \tilde{2}}^{\nu \sigma \delta} \eta_{\lambda \delta} p_{1 \mu} p_{2 \nu} \tilde{p}_{1 \rho} \tilde{p}_{2 \sigma}\right] G_{1 \tilde{1} ; 2 \tilde{2}} \\
& \text { monर }=\frac{1-\xi}{2} \frac{i}{\hbar}(2 g)^{6} \int d \hat{\tau}_{12 \tilde{1} \tilde{2}} f^{a d e} f^{b c e} c_{1 a} c_{2 b} \tilde{c}_{1 c} \tilde{c}_{2 d}\left[\frac{1}{4} V_{1 \tilde{2}}^{\mu \sigma \lambda} V_{2 \tilde{1}}^{\nu \rho \delta} \eta_{\lambda \delta} p_{1 \mu} p_{2 \nu} \tilde{p}_{1 \rho} \tilde{p}_{2 \sigma}\right] G_{1 \tilde{2} ; 2 \tilde{1}}
\end{aligned}
$$

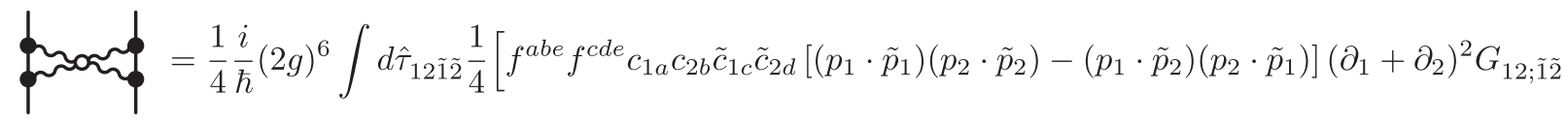

$$
\begin{aligned}
& +2 \rho f^{a c e} f^{b d e} c_{1 a} c_{2 b} \tilde{c}_{1 c} \tilde{c}_{2 d}\left[\left(p_{1} \cdot p_{2}\right)\left(\tilde{p}_{1} \cdot \tilde{p}_{2}\right)-\left(p_{1} \cdot \tilde{p}_{2}\right)\left(p_{2} \cdot \tilde{p}_{1}\right)\right]\left(\partial_{1}+\tilde{\partial}_{1}\right)^{2} G_{1 \tilde{1} ; 2 \tilde{2}} \\
& \left.+2(1-\rho) f^{a d e} f^{b c e} c_{1 a} c_{2 b} \tilde{c}_{1 c} \tilde{c}_{2 d}\left[\left(p_{1} \cdot p_{2}\right)\left(\tilde{p}_{1} \cdot \tilde{p}_{2}\right)-\left(p_{1} \cdot \tilde{p}_{1}\right)\left(p_{2} \cdot \tilde{p}_{2}\right)\right]\left(\partial_{1}+\tilde{\partial}_{2}\right)^{2} G_{1 \tilde{2} ; 2 \tilde{1}}\right]
\end{aligned}
$$

where we have introduced the two-loop function $G_{12 ; \tilde{1} \tilde{2}}=\int d^{4} x d^{4} y D_{1 x} D_{2 x} D_{x y} D_{\tilde{1} y} D_{\tilde{2} y}$. Note that the diagrams (17) and (18) are numerically identical under a relabeling of the worldline variables $\tilde{\tau}_{i}$. However, they will lead to different double copies. This ambiguity is captured by introducing the parameter $\xi$. The same applies to the last two lines of Eq. (19), whose ambiguity we parametrize with $\rho$. Of course, the right choice should be dictated by requiring the color-kinematic algebra, i.e., kinematical Jacobi identity, to hold for the numerators - thereby possibly adding vanishing terms to these expressions, reflecting generalized gauge transformations [27]. We will postpone this analysis to the post-Newtonian limit in the next section.

The final group of bulk diagrams is nonsymmetric in the two worldlines and carries the color structures $[c, c]^{a}[c, \tilde{c}]^{a}$ and mirrors $[c, \tilde{c}]^{a}[\tilde{c}, \tilde{c}]^{a}$. They also contribute to $2 \mathrm{PN}$ and read

$$
\begin{aligned}
& \text { ફૅ } \\
& +\alpha_{2} f^{a c e} f^{b d e} c_{1 a} c_{2 b} c_{3 c} \tilde{c}_{1 d} V_{13}^{\mu \rho \lambda} V_{2 \tilde{1}}^{\nu \sigma \delta} \eta_{\lambda \delta} p_{1 \mu} p_{2 \nu} p_{3 \rho} \tilde{p}_{1 \sigma} G_{13 ; 2 \tilde{1}} \\
& \left.+\left(3-\alpha_{1}-\alpha_{2}\right) f^{a d e} f^{b c e} c_{1 a} c_{2 b} c_{3 c} \tilde{c}_{1 d} V_{1 \tilde{1}}^{\mu \sigma \lambda} V_{23}^{\nu \rho} \eta_{\lambda \delta} p_{1 \mu} p_{2 \nu} p_{3 \rho} \tilde{p}_{1 \sigma} G_{1 \tilde{1} ; 23}\right]
\end{aligned}
$$

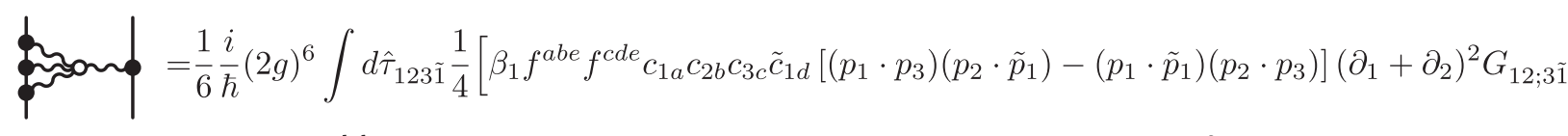

$$
\begin{aligned}
& +\beta_{2} f^{a c e} f^{b d e} c_{1 a} c_{2 b} c_{3 c} \tilde{c}_{1 d}\left[\left(p_{1} \cdot p_{2}\right)\left(p_{3} \cdot \tilde{p}_{1}\right)-\left(p_{1} \cdot \tilde{p}_{1}\right)\left(p_{2} \cdot p_{3}\right)\right]\left(\partial_{1}+\partial_{3}\right)^{2} G_{13 ; 2 \tilde{1}} \\
& \left.+\left(3-\beta_{1}-\beta_{2}\right) f^{a d e} f^{b c e}{ }_{c_{1 a}} c_{2 b} c_{3 c} \tilde{c}_{1 d}\left[\left(p_{1} \cdot p_{2}\right)\left(p_{3} \cdot \tilde{p}_{1}\right)-\left(p_{1} \cdot p_{3}\right)\left(p_{2} \cdot \tilde{p}_{1}\right)\right]\left(\partial_{1}+\tilde{\partial}_{1}\right)^{2} G_{1 \tilde{1} ; 32}\right] \text {. }
\end{aligned}
$$

Again, we use $\alpha_{1}, \alpha_{2}, \beta_{1}, \beta_{2}$ to parametrize the ambiguity of relabeling the worldline variables $\tau_{i}$. Naturally, there is also the set of mirrored graphs swapping tilded and nontilded quantities.

\section{DOUBLE-COPY PRESCRIPTION AT NNLO}

In order to clearly spell out the double-copy prescription for NNLO that we applied we do this in greater detail for the first group of diagrams with color structure $(c \cdot \tilde{c})^{3}$ from Eqs. (10)-(12). The sum of these graphs takes the form 


$$
\mathcal{M}_{\mathrm{YM}}^{(c \cdot \tilde{c})^{3}}=\frac{1}{6}\left(\frac{i}{\hbar}\right)^{3}(2 g)^{6} \int d \hat{\tau}_{123 \tilde{1} \tilde{2} \tilde{3}} N_{(c \cdot \tilde{c})^{3}} C_{(c \cdot \tilde{c})^{3}} D_{1 \tilde{1}} D_{2 \tilde{2}} D_{3 \tilde{3}}
$$

with the color factor $C_{(c \cdot \tilde{c})^{3}}=\left(c_{1} \cdot \tilde{c}_{1}\right)\left(c_{2} \cdot \tilde{c}_{2}\right)\left(c_{3} \cdot \tilde{c}_{3}\right)$ and the kinematic numerator

$$
\begin{aligned}
N_{(c \cdot \tilde{c})^{3}=} & \left(p_{1} \cdot \tilde{p}_{1}\right)\left(p_{2} \cdot \tilde{p}_{2}\right)\left(p_{3} \cdot \tilde{p}_{3}\right) \\
& +\frac{1}{2} \frac{\hbar}{i}\left[\frac{\delta\left(\tau_{1}-\tau_{2}\right)}{\lambda_{2}}\left(\tilde{p}_{1} \cdot \tilde{p}_{2}\right)\left(p_{3} \cdot \tilde{p}_{3}\right)+\operatorname{cyclic}(1,2,3)+\frac{\delta\left(\tilde{\tau}_{1}-\tilde{\tau}_{2}\right)}{\tilde{\lambda}_{2}}\left(p_{1} \cdot p_{2}\right)\left(p_{3} \cdot \tilde{p}_{3}\right)+\operatorname{cyclic}(\tilde{1}, \tilde{2}, \tilde{3})\right] \\
& +\frac{3}{2}\left(\frac{\hbar}{i}\right)^{2} \frac{\delta\left(\tau_{1}-\tau_{2}\right)}{\lambda_{2}} \frac{\delta\left(\tilde{\tau}_{3}-\tilde{\tau}_{2}\right)}{\tilde{\lambda}_{2}}\left(p_{2} \cdot \tilde{p}_{1}\right) .
\end{aligned}
$$

The double copy is then performed by replacing $C_{(c \cdot \tilde{c})^{3}} \rightarrow N_{(c \cdot \tilde{c})^{3}}$ and $2 g \rightarrow i \kappa$ in Eq. (22) thereby constructing the putative dilaton-gravity contribution $\mathcal{M}_{\mathrm{dg}}^{(c \cdot \tilde{c})^{3}}$ from Yang-Mills theory. The obtained expressions indeed exponentiate:

$$
\begin{aligned}
\mathcal{M}_{\mathrm{dg}}^{(c \cdot \tilde{c})^{3}}= & \exp \left[\frac{i}{\hbar} \frac{(i \kappa)^{2}}{2} \int d \hat{\tau}_{1 \tilde{2}}\left(p_{1} \cdot \tilde{p}_{2}\right)^{2} D_{1 \tilde{2}}+\frac{i}{\hbar} \frac{(i \kappa)^{4}}{2} \int d \hat{\tau}_{1 \tilde{2} \tilde{3}}\left(p_{1} \cdot \tilde{p}_{2}\right)\left(p_{1} \cdot \tilde{p}_{3}\right)\left(\tilde{p}_{2} \cdot \tilde{p}_{3}\right) D_{1 \tilde{2}} D_{1 \tilde{3}}\right. \\
& +\frac{i}{\hbar} \frac{(i \kappa)^{6}}{2} \int d \hat{\tau}_{12 \tilde{1} \tilde{2}} \frac{1}{2}\left[\left(p_{1} \cdot \tilde{p}_{1}\right)\left(p_{2} \cdot \tilde{p}_{2}\right)\left(p_{1} \cdot \tilde{p}_{2}\right)\left(p_{2} \cdot \tilde{p}_{1}\right)+\left(p_{1} \cdot \tilde{p}_{1}\right)\left(p_{2} \cdot \tilde{p}_{2}\right)\left(p_{1} \cdot p_{2}\right)\left(\tilde{p}_{1} \cdot \tilde{p}_{2}\right)\right] D_{1 \tilde{1}} D_{2 \tilde{2}} D_{2 \tilde{1}} \\
& \left.+\frac{i}{\hbar}(i \kappa)^{6} \int d \hat{\tau}_{1 \tilde{1} \tilde{2} \tilde{3}} \frac{1}{4}\left(p_{1} \cdot \tilde{p}_{1}\right)\left(p_{1} \cdot \tilde{p}_{3}\right)\left(\tilde{p}_{1} \cdot \tilde{p}_{3}\right)\left(\tilde{p}_{2} \cdot \tilde{p}_{3}\right) D_{1 \tilde{1}} D_{1 \tilde{2}} D_{1 \tilde{3}}+(\operatorname{mirrored})+\mathcal{O}\left(\hbar^{0}\right)\right]\left.\right|_{\kappa^{6}} \cdot
\end{aligned}
$$

Note that the first and third terms in the above are mirror symmetric, hence the factor of $\frac{1}{2}$. Importantly also the suppressed quantum terms at $\mathcal{O}\left(\hbar^{0}\right)$ exponentiate, which is only true if one symmetrizes all the $\tau_{i}$ integrands in the YM representation. They contain ill-defined expressions proportional to $\delta(0)$. We hence consistently recover the exponentiated LO and NLO order results of [59] at this order in $\kappa^{6}$. The contributions at NNLO to the effective action from this sector are thus given by the last two lines in Eq. (25). Performing the double copy of the other groups of graphs, i.e., the symmetric bulk graphs stemming from Eqs. (16)-(19) as well as the nonsymmetric bulk graphs arising from Eqs. (20)-(21), proceeds along the same lines. Under the double copy one then produces the NNLO topologies displayed in Fig. 2. They indeed match the topologies present in gravity at the $2 \mathrm{PN}$ order,

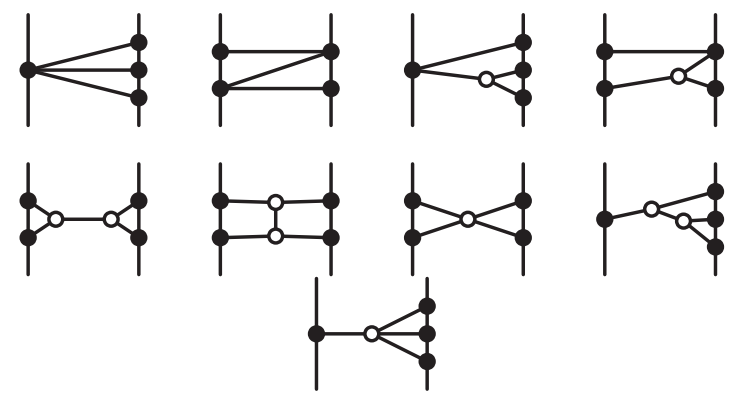

FIG. 2. Topologies generated by the double copy at NNLO. see, e.g., [63]. The total effective action at 3PM order produced via the double copy would follow by performing the worldline and bulk integrals. We shall, however, proceed to work out the $2 \mathrm{PN}$ expansion whose integrals are more straightforward to obtain.

Finally, we remark that the double-copy prescription that we applied here, notably the rewriting of the worldline-bulk vertices in trivalent ones using $\delta$-functions, also follows from a systematic treatment employing the bifundamental scalar field theory as the seed to define the kinematic numerators and denominators. This route was advocated for in [58] for the analogous radiation problem at the level of the equations of motion. This fact essentially hinges on the simple observation that the worldline only interacts linearly with the bulk fields in bifundamental scalar field theory also in the first-order worldline formalism.

\section{PN EXPANSION AND KINEMATICAL JACOBI IDENTITY}

Details of performing the PN expansion were discussed in [59]. The expansion combines a weak-field and slow-motion approximation for bound binaries. The virial theorem in this setup states

$$
\frac{\mathbf{v}_{r}^{2}}{c^{2}} \sim \frac{\kappa^{2}(m+\tilde{m})}{c^{2} 32 \pi r}
$$

where $r=|\mathbf{x}-\tilde{\mathbf{x}}|$ is the distance and $\mathbf{v}_{r}$ the relative velocity of the two particles, with the speed of light $c$ 
restored. Just as in [59] the post-Newtonian expansion parameter is $c^{-1}$ and the $2 \mathrm{PN}$ order amounts to the order $c^{-6}$. We note the scaling properties

$$
\begin{aligned}
\left(p_{\mu}\right)=(E,-\mathbf{p}) & \sim\left(\mathcal{O}\left(c^{0}\right), \mathcal{O}\left(c^{-1}\right)\right), \\
\kappa & \sim \mathcal{O}\left(c^{-1}\right), \quad \lambda \sim \mathcal{O}\left(c^{0}\right), \quad \partial_{t} \sim \mathcal{O}\left(c^{-1}\right) .
\end{aligned}
$$

The post-Newtonian expansion of the propagator becomes local in time and is expanded as

$$
\begin{aligned}
D(x) & =\int \frac{d^{4} k}{(2 \pi)^{4}} \frac{e^{-i k_{\mu} x^{\mu}}}{k_{\mu} k^{\mu}+i \epsilon} \\
& =-\int \frac{d^{3} \mathbf{k}}{(2 \pi)^{3}} \frac{e^{i \mathbf{k} \cdot \mathbf{x}}}{\mathbf{k}^{2}}\left[1-\frac{\partial_{t}^{2}}{\mathbf{k}^{2}}+\frac{\partial_{t}^{4}}{\mathbf{k}^{4}}+\cdots\right] \delta(t) .
\end{aligned}
$$

The diagrams with the color factor $(c \cdot \tilde{c})^{3}$ have a contribution to the double-copy effective action and are extracted from the last three terms in Eq. (25). We further eliminate the Lagrange multipliers $\lambda_{i}, \tilde{\lambda}_{i}$ and worldline energies $E_{i}, \tilde{E}_{i}$ using their equations of motion. At $2 \mathrm{PN}$, we only need the leading-order terms, and we get

$$
L_{\mathrm{eff}}^{(c \cdot \tilde{c})^{3}}=\frac{32 G^{3} m^{2} \tilde{m}^{2}}{r^{3}}+\frac{8 G^{3} m^{3} \tilde{m}}{r^{3}}+\frac{8 G^{3} m \tilde{m}^{3}}{r^{3}},
$$

where we have also replaced $\kappa$ with $\sqrt{32 \pi G}$.

Similarly, the symmetric bulk graphs from Eqs. (16)-(19) yield

$\frac{(i \kappa)^{6}}{4} \int d \hat{\tau}_{12 \tilde{1} \tilde{2}}\left(N_{s}^{2} G_{12 ; \tilde{1} \tilde{2}}+N_{t}^{2} G_{1 \tilde{1} ; 2 \tilde{2}}+N_{u}^{2} G_{1 \tilde{2} ; 2 \tilde{1}}\right)$,

where

$$
\begin{gathered}
N_{s}=\frac{1}{4} p_{1 \mu} p_{2 \nu} \tilde{p}_{1 \rho} \tilde{p}_{2 \sigma}\left[V_{12}^{\mu \nu \lambda} V_{\tilde{1} \tilde{2}}^{\rho \sigma \delta} \eta_{\lambda \delta}\right. \\
\left.+\left(\eta^{\mu \rho} \eta^{\nu \sigma}-\eta^{\mu \sigma} \eta^{\nu \rho}\right)\left(\partial_{1}+\partial_{2}\right)^{2}\right], \\
N_{t}=\frac{1}{4} p_{1 \mu} p_{2 \nu} \tilde{p}_{1 \rho} \tilde{p}_{2 \sigma}\left[2 \xi V_{1 \tilde{1}}^{\mu \rho \lambda} V_{2 \tilde{2}}^{\nu \sigma \delta} \eta_{\lambda \delta}\right. \\
\left.+2 \rho\left(\eta^{\mu \nu} \eta^{\rho \sigma}-\eta^{\mu \sigma} \eta^{\nu \rho}\right)\left(\partial_{1}+\tilde{\partial}_{1}\right)^{2}\right], \\
N_{u}=\frac{1}{4} p_{1 \mu} p_{2 \nu} \tilde{p}_{1 \rho} \tilde{p}_{2 \sigma}\left[2(1-\xi) V_{1 \tilde{2}}^{\mu \sigma \lambda} V_{2 \tilde{1}}^{\nu \rho \delta} \eta_{\lambda \delta}\right. \\
\left.+2(1-\rho)\left(\eta^{\mu \nu} \eta^{\rho \sigma}-\eta^{\mu \rho} \eta^{\nu \sigma}\right)\left(\partial_{1}+\tilde{\partial}_{2}\right)^{2}\right] .
\end{gathered}
$$

In the PN limit Eq. (31) reduces to $N_{s}=\frac{1}{4} E_{1} E_{2} \tilde{E}_{1} \tilde{E}_{2}\left(\partial_{1}-\right.$ $\left.\partial_{2}\right) \cdot\left(\tilde{\partial}_{1}-\tilde{\partial}_{2}\right)+\mathcal{O}\left(c^{-1}\right)$ at leading order, i.e., the last two terms in Eq. (31) do not contribute at this order. Similar reductions apply to $N_{t}$ and $N_{u}$, with an extra factor $2 \xi$ in $N_{t}$ and $2(1-\xi)$ in $N_{u}$. The surviving terms may indeed be made to obey the kinematical Jacobi identity at the PN level through a suitable choice of the parameter $\xi$. One has

$$
\begin{aligned}
N_{s}- & N_{t}+N_{u} \\
= & \frac{1}{4} E_{1} E_{2} \tilde{E}_{1} \tilde{E}_{2} \times\left[2(1-\xi)\left(\partial_{1}-\tilde{\partial}_{2}\right) \cdot\left(\partial_{2}-\tilde{\partial}_{1}\right)\right. \\
& \left.-2 \xi\left(\partial_{1}-\tilde{\partial}_{1}\right) \cdot\left(\partial_{2}-\tilde{\partial}_{2}\right)+\left(\partial_{1}-\partial_{2}\right) \cdot\left(\tilde{\partial}_{1}-\tilde{\partial}_{2}\right)\right] \\
& +\mathcal{O}\left(c^{-1}\right),
\end{aligned}
$$

dropping subleading terms at $\mathcal{O}\left(c^{-1}\right)$. Demanding the vanishing of the above relations fixes $\xi=\frac{1}{2}$. The second introduced parameter $\rho$ for the symmetric bulk graphs remains unfixed at the $2 \mathrm{PN}$ level. In fact one checks that the higher-order terms in $c^{-1}$ pertaining to the PM level cannot be made to vanish for any choice of $\rho$. Hence, it appears necessary to add generalized gauge transformationlike terms at the 3PM level, which would, however, nont affect the 2PN level considered here, as is shown in the Appendix.

Hence, we take $\xi=\frac{1}{2}$ and perform the resulting two-loop integrals for the $G_{12 ; 34}$ functions in Eq. (30) using TARCER $[64]$ and FeynCalc $[65,66]$ to find the contribution

$$
L_{\mathrm{eff}}^{\mathrm{sym}}=\frac{8 G^{3} m^{2} \tilde{m}^{2}}{r^{3}}
$$

Turning to the nonsymmetric bulk graphs of Eqs. (20) and (21) we have

$$
\begin{aligned}
& \frac{(i \kappa)^{6}}{6} \int d \hat{\tau}_{123 \tilde{1}} N_{s^{\prime}}^{2} G_{12 ; 3 \tilde{1}}+N_{t^{\prime}}^{2} G_{13 ; 2 \tilde{1}} \\
& \quad+N_{u^{\prime}}^{2} G_{1 \tilde{1} ; 23}+\text { (mirrored) },
\end{aligned}
$$

where

$$
\begin{aligned}
N_{s^{\prime}}= & \frac{1}{4} p_{1 \mu} p_{2 \nu} p_{3 \rho} p_{\tilde{1} \sigma}\left[\alpha_{1} V_{12}^{\mu \nu \lambda} V_{3 \tilde{1}}^{\rho \sigma \delta} \eta_{\lambda \delta}\right. \\
& \left.+\beta_{1}\left(\eta^{\mu \rho} \eta^{\nu \sigma}-\eta^{\mu \sigma} \eta^{\nu \rho}\right)\left(\partial_{1}+\partial_{2}\right)^{2}\right]
\end{aligned}
$$

$N_{t^{\prime}}$ and $N_{u^{\prime}}$ have similar expressions that can be read off from Eqs. (20) and (21). The above argument for the kinematical Jacobi identity of the symmetric bulk graphs also applies to the nonsymmetric graphs. This fixes $\alpha_{1,2}=1$ and leaves $\beta_{1,2}$ arbitrary. In the leading PN limit of Eq. (36) again the terms arising from the quartic vertex cancel leaving us with

$$
L_{\mathrm{eff}}^{\mathrm{nonsym}}=-\frac{4 G^{3} m^{3} \tilde{m}}{3 r^{3}}-\frac{4 G^{3} m \tilde{m}^{3}}{3 r^{3}}
$$

after performing the integrals. Together with the diagrams up to 2PM, already given in [59] but now expanded to $2 \mathrm{PN}$, we may assemble the double-copy prediction for the 
effective action. We further solve the equations of motion for $\lambda_{i}, \tilde{\lambda}_{i}$ and $E_{i}, \tilde{E}_{i}$ order by order to rewrite the action in terms of the worldline coordinates and their derivatives. The resulting expression for the effective action may now be compared to the known result due to Mirshekari and Will [61], suitably adjusting their parameters. For a comparison it is, however, important to realize that the effective action itself is gauge variant and subject to possible field redefinitions. Applying field redefinitions and adding total derivatives, in order to match the velocitydependent terms up to 2PN with the result of [61] we finally find the central result of this work:

$$
\begin{aligned}
L_{\text {eff }}= & -m-\tilde{m}+\frac{1}{2} m \mathbf{v}^{2}+\frac{1}{2} \tilde{m} \tilde{\mathbf{v}}^{2}+\frac{2 G m \tilde{m}}{r}+\frac{1}{8} m \mathbf{v}^{4}+\frac{1}{8} \tilde{m} \tilde{\mathbf{v}}^{4}-\frac{2 G^{2} m \tilde{m}(m+\tilde{m})}{r^{2}} \\
& +\frac{G m \tilde{m}}{r}\left[\mathbf{v}^{2}+\tilde{\mathbf{v}}^{2}-3 \mathbf{v} \cdot \tilde{\mathbf{v}}-(\mathbf{n} \cdot \mathbf{v})(\mathbf{n} \cdot \tilde{\mathbf{v}})\right]+\frac{1}{16} m \mathbf{v}^{6}+\frac{1}{16} \tilde{m} \tilde{\mathbf{v}}^{6} \\
& +\frac{G m \tilde{m}}{r}\left(\frac{1}{2}(\mathbf{v} \cdot \tilde{\mathbf{v}})^{2}+\frac{3}{4} \tilde{\mathbf{v}}^{4}+\frac{7}{4} \mathbf{v}^{2} \tilde{\mathbf{v}}^{2}+\frac{3}{4} \mathbf{v}^{4}-2 \mathbf{v}^{2}(\mathbf{v} \cdot \tilde{\mathbf{v}})-2 \tilde{\mathbf{v}}^{2}(\mathbf{v} \cdot \tilde{\mathbf{v}})\right. \\
& \left.-\frac{3}{4} \mathbf{v}^{2}(\mathbf{n} \cdot \tilde{\mathbf{v}})^{2}-\frac{3}{4} \tilde{\mathbf{v}}^{2}(\mathbf{n} \cdot \mathbf{v})^{2}+\frac{3}{4}(\mathbf{n} \cdot \mathbf{v})^{2}(\mathbf{n} \cdot \tilde{\mathbf{v}})^{2}+(\mathbf{v} \cdot \tilde{\mathbf{v}})(\mathbf{n} \cdot \mathbf{v})(\mathbf{n} \cdot \tilde{\mathbf{v}})\right) \\
& +G m \tilde{m}\left(\frac{3}{4} \tilde{\mathbf{v}}^{2} \mathbf{a} \cdot \mathbf{n}-\frac{1}{4}(\mathbf{a} \cdot \mathbf{n})(\mathbf{n} \cdot \tilde{\mathbf{v}})^{2}-\frac{3}{2}(\mathbf{a} \cdot \tilde{\mathbf{v}})(\mathbf{n} \cdot \tilde{\mathbf{v}})-\frac{3}{4} \mathbf{v}^{2} \tilde{\mathbf{a}} \cdot \mathbf{n}+\frac{1}{4}(\tilde{\mathbf{a}} \cdot \mathbf{n})(\mathbf{n} \cdot \mathbf{v})^{2}+\frac{3}{2}(\mathbf{n} \cdot \mathbf{v})(\tilde{\mathbf{a}} \cdot \mathbf{v})\right) \\
& +\frac{G^{2} m \tilde{m}}{r^{2}}\left(5 m(\mathbf{n} \cdot \mathbf{v})^{2}+m(\mathbf{n} \cdot \tilde{\mathbf{v}})^{2}-4 m(\mathbf{n} \cdot \mathbf{v})(\mathbf{n} \cdot \tilde{\mathbf{v}})+3 m \mathbf{v} \cdot \tilde{\mathbf{v}}-2 m \mathbf{v}^{2}\right. \\
& \left.+5 \tilde{m}(\mathbf{n} \cdot \tilde{\mathbf{v}})^{2}+\tilde{m}(\mathbf{n} \cdot \mathbf{v})^{2}-4 \tilde{m}(\mathbf{n} \cdot \mathbf{v})(\mathbf{n} \cdot \tilde{\mathbf{v}})+3 \tilde{m} \mathbf{v} \cdot \tilde{\mathbf{v}}-2 \tilde{m} \tilde{\mathbf{v}}^{2}\right) \\
& +G^{3}\left(\frac{14 m^{3} \tilde{m}}{3 r^{3}}+\frac{26 m^{2} \tilde{m}^{2}}{r^{3}}+\frac{14 m \tilde{m}^{3}}{3 r^{3}}\right) .
\end{aligned}
$$

Indeed, the 2PN static terms are seen to differ from the result $L_{\text {eff }}^{M W}$ of Mirshekari and Will [61] by $\Delta L_{\text {eff }}:=$ $L_{\text {eff }}-L_{\mathrm{eff}}^{M W},{ }^{1}$

$$
\Delta L_{\mathrm{eff}}=\frac{2 G^{3} m \tilde{m}(m+\tilde{m})^{2}}{r^{3}} .
$$

This disagreement cannot be removed by either field redefinition or adding total derivatives. It is conceivable that an adjustment of the gauge fixing condition in the original Yang-Mills theory (at higher orders in $g$ ), which would modify the double-copy result, could remedy this disagreement. The important point here is that the effective action is a gauge variant quantity. However, such a remedy would question the usefulness of the double-copy procedure.

We have also performed an independent check of the validity of the effective potential of [61] by performing a probe limit. Here one takes $m \ll \tilde{m}$ and compares to the potential experienced by a test particle of mass $m$ in the JNW solution of dilaton-gravity [62] which is the relevant black hole solution in this setup as shown in [48]. We found agreement with the static terms linear in $m$ of [61] and

\footnotetext{
${ }^{1}$ Their parameters are adjusted as $\alpha=1, \bar{\gamma}=-1, \bar{\beta}_{1,2}=0$, $\bar{\delta}_{1,2}=\frac{1}{4}, \bar{\chi}_{1,2}=0$. Also note that converting to our convention needs to take $G^{\mathrm{MW}} \rightarrow 2 G$.
}

disagreement with our double-copy results in Eq. (39). On top we also performed a full perturbative computation in dilaton-gravity at $2 \mathrm{PN}$ order of the static terms again reproducing [61]. In summary, the breakdown of the double-copy procedure for the effective potential is therefore on firm ground.

\section{ACKNOWLEDGMENTS}

We thank Lance Dixon and Wadim Wormsbecher for discussions and comments. This project has received funding from the European Union's Horizon 2020 research and innovation program under Marie Sklodowska-Curie Grant Agreement No. 764850, and the German Research Foundation through Grant No. PL457/3-1.

\section{APPENDIX: IMPLEMENTING THE KINEMATIC JACOBI IDENTITY AT THE 3PM LEVEL}

In Sec. V we showed that the kinematic numerators of the symmetric bulk graphs as well as the nonsymmetric ones obey the kinematic Jacobi identities only at the leading order in the PN limit, $N_{s}-N_{t}+N_{u}=\mathcal{O}\left(c^{-1}\right)$. We shall now construct an explicit representation of these bulk graphs that obeys the Jacobi identity by a generalized gauge transformation [27] and prove that it will not affect the $2 \mathrm{PN}$ answer (39) above. 
After a Fourier transform of the bulk degrees of freedom the symmetric graphs in Yang-Mills theory equations (16)-(19) take the form

$$
\begin{aligned}
I^{\mathrm{sym}}= & \int d^{16} \hat{q} \int d \hat{\tau}_{12 \tilde{1} \tilde{2}}\left[\frac{c_{s} N_{s}}{s}+\frac{c_{t} N_{t}}{t}+\frac{c_{u} N_{u}}{u}\right] \\
& \times \frac{1}{q_{1}^{2} q_{2}^{2} q_{\tilde{1}}^{2} q_{\tilde{2}}^{2}},
\end{aligned}
$$

with $s=\left(q_{1}+q_{2}\right)^{2}, t=\left(q_{1}+q_{\tilde{1}}\right)^{2}, u=\left(q_{1}+q_{\tilde{2}}\right)^{2}$,

$$
d^{16} \hat{q}=\prod_{i=1,2, \tilde{1}, \tilde{2}} d^{4} q_{i} e^{i q_{i} \cdot x_{i}}
$$

and

$$
\begin{aligned}
c_{s} & =f^{a b e} f^{c d e} c_{1 a} c_{2 b} \tilde{c}_{1 c} \tilde{c}_{2 d}, \\
c_{t} & =f^{a c e} f^{b d e} c_{1 a} c_{2 b} \tilde{c}_{1 c} \tilde{c}_{2 d}, \\
c_{u} & =f^{a b e} f^{c d e} c_{1 a} c_{2 b} \tilde{c}_{1 c} \tilde{c}_{2 d} .
\end{aligned}
$$

Moreover, the kinematical numerators $N_{s, t, u}$ in the above are given by the expressions (31)-(33) with the substitution $\partial_{i} \rightarrow i q_{i}$. Clearly, the color factors obey the Jacobi identity

$$
c_{s}-c_{t}+c_{u}=0 .
$$

However, the kinematical numerators fail to fulfill this identity,

$$
N_{s}-N_{t}+N_{u}=\Delta\left(p_{i}, q_{i}\right),
$$

with a lengthy expression $\Delta\left(p_{i}, q_{i}\right)$ following from Eqs. (31)-(33). We now introduce the generalized gauge transformations

$$
\tilde{N}_{a}=N_{a}+\Delta_{a}, \quad a=s, t, u,
$$

which need to obey two conditions: The first one is

$$
\Delta_{s}-\Delta_{t}+\Delta_{u}=-\Delta,
$$

securing the Jacobi identity for the $\tilde{N}_{a}$. The second condition demands to leave $I^{\text {sym }}$ invariant

$$
\begin{aligned}
0= & \int d^{16} \hat{q} \int d \hat{\tau}_{12 \tilde{1} \tilde{2}}\left(c_{s}\left[\frac{\Delta_{s}}{s}+\frac{\Delta_{t}}{t}\right]+c_{u}\left[\frac{\Delta_{u}}{u}+\frac{\Delta_{t}}{t}\right]\right) \\
& \times \frac{1}{q_{1}^{2} q_{2}^{2} q_{\tilde{1}}^{2} q_{\tilde{2}}^{2}},
\end{aligned}
$$

having replaced $c_{t}=c_{s}+c_{u}$ with Eq. (A4). Demanding the vanishing of this at the integrand level yields the simple solutions

$$
\Delta_{s}=-\frac{s \Delta}{s+t+u}, \quad \Delta_{t}=\frac{t \Delta}{s+t+u}, \quad \Delta_{u}=-\frac{u \Delta}{s+t+u} .
$$

Of course, the vanishing of Eq. (A8) would in principle also allow for total derivative contributions in the integrands. However, all solutions will lead to identical double-copy results so it is sufficient to stick with the simple ones of Eq. (A9). Now as $\Delta=\mathcal{O}\left(c^{-1}\right)$-as was shown in Eq. (34) for the choice $\xi=\frac{1}{2}$ and the fact that $s, t, u$ are independent of $c$ or $\kappa$ we immediately conclude that the kinematical Jacobi relation respecting numerators $\tilde{N}_{a}$ differs from the original $N_{a}$ only by terms of order $\mathcal{O}\left(c^{-1}\right)$. This implies in turn that the contributions from the double copied symmetric graphs to the putative effective action of dilaton gravity obtained from the $\tilde{N}_{a}$ does not differ from the one constructed by the $N_{a}$ computed in Eq. (39) at the 2PN level,

$$
\begin{aligned}
& \int d \hat{\tau}_{12 \tilde{1} \tilde{2}}\left(\tilde{N}_{s}^{2} G_{12 ; \tilde{1} \tilde{2}}+\tilde{N}_{t}^{2} G_{1 \tilde{1} ; 2 \tilde{2}}+\tilde{N}_{u}^{2} G_{1 \tilde{2} ; 2 \tilde{1}}\right) \\
& =\int d \hat{\tau}_{12 \tilde{1} \tilde{2}}\left(N_{s}^{2} G_{12 ; \tilde{1} \tilde{2}}+N_{t}^{2} G_{1 \tilde{1} ; 2 \tilde{2}}+N_{u}^{2} G_{1 \tilde{2} ; 2 \tilde{1}}\right) \\
& \quad+\mathcal{O}\left(c^{-1}\right) .
\end{aligned}
$$

The analogous argument goes through for the nonsymmetric bulk graphs.

In summary, we have thus shown that implementing the kinematical Jacobi identity at the 3PM level does not affect the $2 \mathrm{PN}$ results reported in the main text.
[1] B. P. Abbott et al. (Virgo and LIGO Scientific Collaborations), Phys. Rev. Lett. 116, 061102 (2016).

[2] L. Blanchet, Living Rev. Relativity 17, 2 (2014).

[3] G. Schäfer and P. Jaranowski, Living Rev. Relativity 21, 7 (2018).
[4] T. Futamase and Y. Itoh, Living Rev. Relativity 10, 2 (2007).

[5] T. Ledvinka, G. Schaefer, and J. Bicak, Phys. Rev. Lett. 100, 251101 (2008).

[6] K. Westpfahl, Fortschr. Phys. 33, 417 (1985). 
[7] W. D. Goldberger and I. Z. Rothstein, Phys. Rev. D 73, 104029 (2006).

[8] S. Foffa, Phys. Rev. D 89, 024019 (2014).

[9] W. D. Goldberger, in Particle Physics and Cosmology: The Fabric of Spacetime, Proceedings of the Les Houches Summer School, Session LXXXVI, edited by F. Bernardeau, C. Grojean, and J. Dalibard (Elsevier, New York, 2006).

[10] R. A. Porto, Phys. Rep. 633, 1 (2016).

[11] M. Levi, arXiv:1807.01699.

[12] T. Damour, P. Jaranowski, and G. Schäfer, Phys. Rev. D 89, 064058 (2014).

[13] T. Damour, P. Jaranowski, and G. Schäfer, Phys. Rev. D 93, 084014 (2016).

[14] L. Bernard, L. Blanchet, A. Bohé, G. Faye, and S. Marsat, Phys. Rev. D 95, 044026 (2017).

[15] T. Marchand, L. Bernard, L. Blanchet, and G. Faye, Phys. Rev. D 97, 044023 (2018).

[16] S. Foffa, P. Mastrolia, R. Sturani, and C. Sturm, Phys. Rev. D 95, 104009 (2017).

[17] S. Foffa, R. A. Porto, I. Rothstein, and R. Sturani, Phys. Rev. D 100, 024048 (2019).

[18] M. Levi and J. Steinhoff, arXiv:1607.04252.

[19] S. Foffa, P. Mastrolia, R. Sturani, C. Sturm, and W. J. Torres Bobadilla, Phys. Rev. Lett. 122, 241605 (2019).

[20] J. Blümlein, A. Maier, and P. Marquard, arXiv:1902.11180.

[21] L. Blanchet, G. Faye, B. R. Iyer, and S. Sinha, Classical Quantum Gravity 25, 165003 (2008); 29, 239501(E) (2012).

[22] A. Buonanno and T. Damour, Phys. Rev. D 59, 084006 (1999).

[23] A. Buonanno and T. Damour, Phys. Rev. D 62, 064015 (2000).

[24] A. Bohé et al., Phys. Rev. D 95, 044028 (2017).

[25] A. Nagar et al., Phys. Rev. D 98, 104052 (2018).

[26] Z. Bern, J. J. M. Carrasco, and H. Johansson, Phys. Rev. D 78, 085011 (2008).

[27] Z. Bern, T. Dennen, Y.-t. Huang, and M. Kiermaier, Phys. Rev. D 82, 065003 (2010).

[28] Z. Bern, J. J. M. Carrasco, and H. Johansson, Phys. Rev. Lett. 105, 061602 (2010).

[29] Z. Bern, H.-H. Chi, L. Dixon, and A. Edison, Phys. Rev. D 95, 046013 (2017).

[30] Z. Bern, J. J. Carrasco, W.-M. Chen, A. Edison, H. Johansson, J. Parra-Martinez, R. Roiban, and M. Zeng, Phys. Rev. D 98, 086021 (2018).

[31] Y. Iwasaki, Prog. Theor. Phys. 46, 1587 (1971).

[32] M. J. Duff, Phys. Rev. D 7, 2317 (1973).

[33] B. R. Holstein and J. F. Donoghue, Phys. Rev. Lett. 93, 201602 (2004).

[34] D. Neill and I. Z. Rothstein, Nucl. Phys. B877, 177 (2013).

[35] N. E. J. Bjerrum-Bohr, J. F. Donoghue, and P. Vanhove, J. High Energy Phys. 02 (2014) 111.

[36] A. Luna, I. Nicholson, D. O'Connell, and C. D. White, J. High Energy Phys. 03 (2018) 044.
[37] N. E. J. Bjerrum-Bohr, P. H. Damgaard, G. Festuccia, L. Platé, and P. Vanhove, Phys. Rev. Lett. 121, 171601 (2018).

[38] D. A. Kosower, B. Maybee, and D. O'Connell, J. High Energy Phys. 02 (2019) 137.

[39] A. Brandhuber and G. Travaglini, arXiv:1905.05657.

[40] W. T. Emond and N. Moynihan, arXiv:1905.08213.

[41] C. Cheung, I. Z. Rothstein, and M. P. Solon, Phys. Rev. Lett. 121, 251101 (2018).

[42] A. Cristofoli, N. E. J. Bjerrum-Bohr, P. H. Damgaard, and P. Vanhove, arXiv:1906.01579.

[43] Z. Bern, C. Cheung, R. Roiban, C.-H. Shen, M. P. Solon, and M. Zeng, Phys. Rev. Lett. 122, 201603 (2019).

[44] T. Damour, Phys. Rev. D 97, 044038 (2018).

[45] T. Damour, Phys. Rev. D 94, 104015 (2016).

[46] R. Monteiro, D. O'Connell, and C. D. White, J. High Energy Phys. 12 (2014) 056.

[47] A. Luna, R. Monteiro, D. O'Connell, and C. D. White, Phys. Lett. B 750, 272 (2015).

[48] A. Luna, R. Monteiro, I. Nicholson, A. Ochirov, D. O'Connell, N. Westerberg, and C. D. White, J. High Energy Phys. 04 (2017) 069.

[49] A. Luna, R. Monteiro, I. Nicholson, and D. O'Connell, Classical Quantum Gravity 36, 065003 (2019).

[50] D. S. Berman, E. Chacón, A. Luna, and C. D. White, J. High Energy Phys. 01 (2019) 107.

[51] M. Carrillo González, B. Melcher, K. Ratliff, S. Watson, and C. D. White, J. High Energy Phys. 07 (2019) 167.

[52] W. D. Goldberger and A. K. Ridgway, Phys. Rev. D 95, 125010 (2017).

[53] W. D. Goldberger and A. K. Ridgway, Phys. Rev. D 97, 085019 (2018).

[54] W. D. Goldberger, J. Li, and S. G. Prabhu, Phys. Rev. D 97, 105018 (2018).

[55] W. D. Goldberger, S. G. Prabhu, and J. O. Thompson, Phys. Rev. D 96, 065009 (2017).

[56] D. Chester, Phys. Rev. D 97, 084025 (2018).

[57] Y. F. Bautista and A. Guevara, arXiv:1903.12419.

[58] C.-H. Shen, J. High Energy Phys. 11 (2018) 162.

[59] J. Plefka, J. Steinhoff, and W. Wormsbecher, Phys. Rev. D 99, 024021 (2019).

[60] T. Damour and G. Esposito-Farese, Classical Quantum Gravity 9, 2093 (1992).

[61] S. Mirshekari and C. M. Will, Phys. Rev. D 87, 084070 (2013).

[62] A. I. Janis, E. T. Newman, and J. Winicour, Phys. Rev. Lett. 20, 878 (1968).

[63] J. B. Gilmore and A. Ross, Phys. Rev. D 78, 124021 (2008).

[64] R. Mertig and R. Scharf, Comput. Phys. Commun. 111, 265 (1998).

[65] R. Mertig, M. Bohm, and A. Denner, Comput. Phys. Commun. 64, 345 (1991).

[66] V. Shtabovenko, R. Mertig, and F. Orellana, Comput. Phys. Commun. 207, 432 (2016). 